\title{
FOREGROUND GALAXIES AROUND LUMINOUS QUASARS
}

\author{
J. VON LINDE, U. BORGEEST AND S. REFSDAL \\ Hamburger Sternwarte \\ Gojenbergsweg 112, D-21029 Hamburg, Germany \\ AND \\ K.-J. SCHRAMM AND E. VAN DROM \\ Université de Liège, Inst. d'Astrophysique \\ 5, Avenue de Cointe, B-4000 Liège, Belgium
}

We compare galaxy counts in deep R-band exposures of the fields of 36 highly luminous, high redshift QSOs to those in control fields at a distance of $1 \mathrm{deg}$. We find indication for a weak overdensity of galaxies in the foreground of QSOs on scales of arcminutes on a low significance level. Counts inside rings around the quasars, stars in the quasar fields and stars in the control fields show evidence for an excess of galaxies on scales of several arcseconds around the quasars as well as for a stronger clustering of galaxies in the QSO fields than in the control fields. We interpret this in terms of an amplification bias by gravitational lensing.

Observations were done at the DSAZ 3.5 and $2.2 \mathrm{~m}$ telescopes at Calar Alto, Spain, the ESO NTT, Chile, and the Nordic Optical Telescope (NOT) at La Palma. Quasars were selected with respect to optical luminosity (12 QSOs, $\mathrm{z} \geq 1 \mathrm{M}_{V} \leq-29.0 ; \mathrm{H}_{0}=50 \mathrm{~km} \mathrm{~s}^{-1} \mathrm{Mpc}^{-1}, \mathrm{q}_{0}=0$ ), and to both optical luminosity and radio flux (24 QSOs, $\mathrm{z} \geq 1.5, \mathrm{M}_{V} \leq-27.0 \mathrm{mag}, \mathrm{S}(6 \mathrm{~cm})$ $\geq 0.8 \mathrm{Jy}$ ). All fields were observed in the Johnson $\mathrm{R}$ band.

Figure 1a shows the galaxy counts in the quasar vs. control fields for 32 objects down to a limiting magnitude of 21.5 rmag (the 4 fields observed at the NOT are not included here because of the small field or a significantly lower brightness limit, respectively). Assuming Poisson statistics, and deriving the variance from the galaxy counts in the respective control fields, we find 6 fields with an excess of galaxies of more than $3 \sigma$ vs. only one field with a significant underdensity. The confidence level, however, is very low, as can be seen by comparison with the distribution of stars in the fields (fig. 1b). The total overdensity of galaxies in the quasar fields is $5 \%$. 

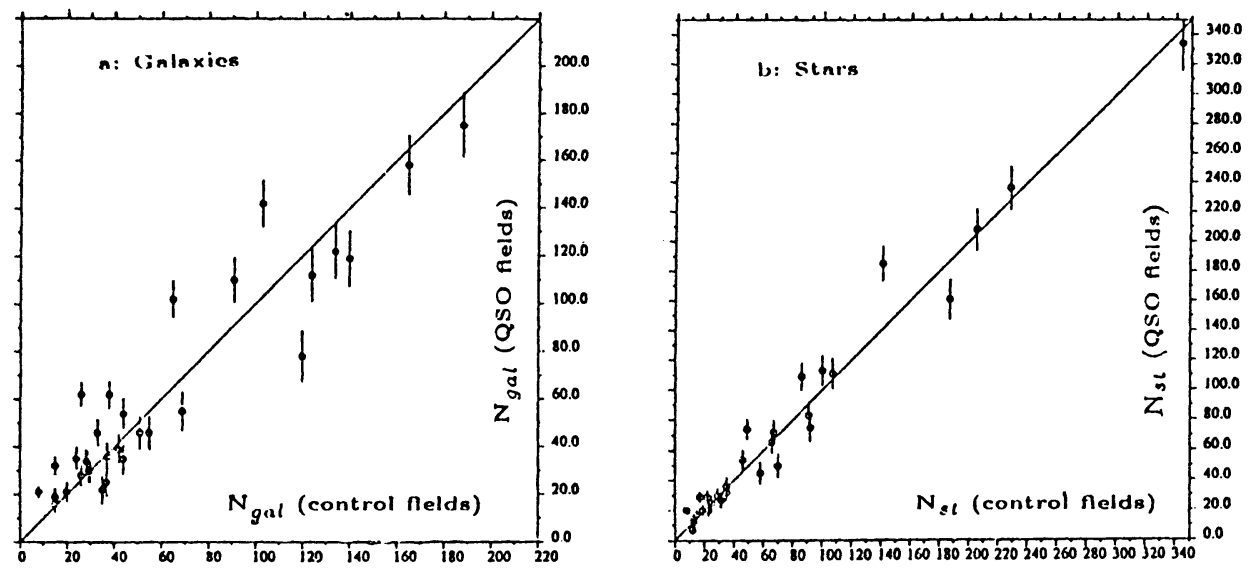

Fig. 1

The results of the 19 fields around radioloud quasars with $S(6 \mathrm{~cm}) \geq 1 \mathrm{Jy}$ show no significant difference to those of the entire sample.

The table below shows the numbers of galaxies in rings of $4^{\prime \prime} \leq r \leq 15^{\prime \prime}$ around the qso $\left(\mathrm{N}_{q s o}^{q f}\right)$, one star in the qso field $\left(\mathrm{N}_{\text {star }}^{q f}\right)$, and two stars in the control fields at respective positions $\left(\mathrm{N}_{s 1}^{c f}, \mathrm{~N}_{s 2}^{c f}\right)$.

\begin{tabular}{l|c|c|c|c|c|c} 
Sample (N) & $\mathrm{N}_{q s o}^{q f}$ & $\mathrm{~N}_{\text {star }}^{q f}$ & $\mathrm{~N}_{s 1}^{c f}$ & $\mathrm{~N}_{s 2}^{c f}$ & $\left\langle\mathrm{~N}_{\text {stars }}>\right.$ & $\mathrm{N}_{q s o} /<\mathrm{N}_{\text {stars }}>$ \\
\hline all (36) & 45 & 20 & 31 & 33 & 24.7 & $1.61 \pm 0.22$ \\
S >1 Jy (19) & 24 & 12 & 20 & 20 & 17.33 & $1.38 \pm 0.34$ \\
opt (8) & 8 & 0 & 3 & 4 & 2.43 & $3.42 \pm 1.8$ \\
\hline
\end{tabular}

Mind that the enhancement factor is even larger, when only the counts inside the QSO fields are taken into account: $\mathrm{N}_{q s o}^{q f} / \mathrm{N}_{\text {star }}^{q f}=2.25$ and 2.0 for the entire sample and the $1 \mathrm{Jy}$-subsample, respectivly. If this is not due to statistical fluctuation it gives indication for clustering of galaxies in the QSO vicinity, since the average number density of galaxies is nearly equal in QSO and control fields.

The results do not give evidence for a magnification bias by clusters of galaxies. However, they show indication for a stronger clustering of galaxies around the quasars than in random fields at the sky. The overdensity of galaxies is weaker in the subsample of extremly radioloud objects. If this is not due to statistical fluctuations, it contradicts the hypothesis of the "multiple waveband bias". A possible explanation would be, that the luminosity function of flat spectrum quasars is not as steep as that of radio quiet objects. 